\title{
RANDOM FIXED POINTS OF MULTIVALUED INWARD RANDOM OPERATORS
}

\author{
A. R. KHAN AND A. A. DOMLO \\ Received 5 June 2006; Revised 26 August 2006; Accepted 27 August 2006
}

The purpose of this paper is to provide a substantial improvement and random analogues of several results due to Benavides and Ramírez (2004). Our work sets random versions of the results of Shahzad and Lone (2005) and improves the work of Plubtieng and Kumam (2006).

Copyright (c) 2006 A. R. Khan and A. A. Domlo. This is an open access article distributed under the Creative Commons Attribution License, which permits unrestricted use, distribution, and reproduction in any medium, provided the original work is properly cited.

\section{Introduction}

Kirk and Massa [7] proved the existence of fixed points for multivalued nonexpansive self-maps in a Banach space $X$ for which the asymptotic center of a bounded sequence in a closed bounded convex subset is nonempty and compact; this occurs, for instance, if $X$ is a uniformly convex space. If $X$ is nearly uniformly convex, then the asymptotic center of a bounded sequence can be a noncompact set (see [8]). This fact motivated Benavides and Ramírez [4] to generalize Kirk-Massa theorem to a class of Banach spaces where the asymptotic center of a sequence is not necessarily a compact set; specifically, they established fixed point theorems for multivalued nonexpansive self-maps in a Banach space whose characteristic of noncompact convexity is less than 1. Benavides and Ramírez [5] obtained results similar to those in [4] for non-self-maps satisfying the inwardness condition. More recently, Shahzad and Lone [12] have replaced nonexpansive maps with $S L$ maps in the results of Benavides and Ramírez [5].

One of the aspects of nonlinear functional analysis is to randomize deterministic fixed point results of the maps. In this regard, we cannot follow the arguments of the proofs for the deterministic cases because these may not be constructive enough to assure the measurability of the random fixed point. Indeed, deterministic results are used as a tool in our proofs to obtain random solutions. In this paper, we prove random fixed point theorems for multivalued inward random operators; in particular, we establish random

Hindawi Publishing Corporation Journal of Applied Mathematics and Stochastic Analysis Volume 2006, Article ID 19428, Pages 1-8

DOI 10.1155/JAMSA/2006/19428 
2 Random fixed points of multivalued inward random operators

analogues of the results of Shahzad and Lone [12]. Our work also extends [11, Theorems 3.2 and 3.3].

\section{Preliminaries}

Let $(X, d)$ be a metric space. We denote by $2^{X}$ (resp., $\left.C(X), C B(X), K(X), K C(X)\right)$ the class of all nonempty subsets (resp., nonempty closed, nonempty closed bound, nonempty compact, nonempty compact convex subsets) of $X$. The diameter of $B \subseteq X$ is given by $\operatorname{diam} B=\sup \{d(x, y): x, y \in B\}$. Let $H$ be the Hausdorff metric with respect to $d$ :

$$
H(A, B)=\max \left\{\sup _{x \in A} d(x, B), \sup _{y \in B} d(y, A)\right\}, \quad \text { for every } A, B \in C(X) .
$$

We recall some definitions.

A Banach space $X$ is said to satisfy Opial's property if for each $x \in X$ and each sequence $\left\{x_{n}\right\}$ weakly convergent to $x$, the following condition holds for all $x \neq y: \liminf _{n \rightarrow \infty} \| x_{n}-$ $x\left\|<\liminf _{n \rightarrow \infty}\right\| x_{n}-y \|$. If $\lim _{n \rightarrow \infty}$ inf $\left\|x_{n}-x\right\| \leq \lim _{n \rightarrow \infty}$ inf $\left\|x_{n}-y\right\|$ holds, then $X$ satisfies the nonstrict Opial's property.

Let $C$ be a nonempty closed subset of a Banach space $X$. A multivalued map $T: C \rightarrow$ $2^{X}$ is (i) upper semicontinuous if $\{x \in C: T x \subset V\}$ is open whenever $V \subset X$ is open; (ii) lower semicontinuous if $T^{-1}(V)=\{x \in C: T x \cap V \neq \phi\}$ is open whenever $V \subset X$ is open; (iii) continuous if it is both upper and lower semicontinuous (see [2, page 299]); (iv) nonexpansive if $H(T x, T y) \leq\|x-y\|$, for all $x, y \in C$.

The Kuratowski, Hausdorff, and separation measures of noncompactness of a nonempty bounded subset $B$ of $X$ are, respectively, defined as the numbers

$$
\begin{gathered}
\alpha(B)=\inf \{r>0: B \text { can be covered by finitely many sets of diameter } \leq r\}, \\
\chi(B)=\inf \{r>0: B \text { can be covered by finitely many balls of radius } \leq r\}, \\
\beta(B)=\sup \{r>0: B \text { has an infinite } r \text {-separation }\} .
\end{gathered}
$$

The map $T: C \rightarrow 2^{X}$ is called $\gamma$-condensing (resp., $1-\gamma$-contractive) where $\gamma=\alpha(\cdot)$ or $\chi(\cdot)$ if, for each bounded subset $B$ of $C$ with $\gamma(B)>0$, the following holds:

$$
\gamma(T(B))<\gamma(B) \quad(\text { resp., } \gamma(T(B)) \leq \gamma(B)), \quad \text { where } T(B)=\bigcup_{x \in B} T x .
$$

Let $X$ be a Banach space and $\phi=\alpha, \beta$, or $\chi$. Then the modulus and characteristic of noncompact convexity associated to $\phi$ are, respectively, defined by

$$
\Delta_{X, \phi}(\epsilon)=\inf \left\{1-d(0, A): A \subset B_{X} \text { is convex, } \phi(A) \geq \epsilon\right\},
$$

where $B_{X}$ is the unit ball of $X$, and

$$
\epsilon_{\phi}(X)=\sup \left\{\epsilon \geq 0: \Delta_{X, \phi}(\epsilon)=0\right\} .
$$

If $\epsilon_{\phi}(X)=0$, then the space $X$ is called nearly uniformly convex.

Note that if $\epsilon_{\alpha}(X)<1$, then $X$ is reflexive (this is also true if $\epsilon_{\phi}(X)<1$ where $\phi=\chi$ or $\beta)$ (see [1, Theorem 5.1.7 and Remark 5.1.7]). 
The inward set of $C$ at $x \in C$ is defined by $I_{C}(x)=\{x+\lambda(y-x): \lambda \geq 0, y \in C\}$. Clearly, $C \subset I_{C}(x)$ and it is not hard to show that $I_{C}(x)$ is a convex set when $C$ is so.

The map $T: C \rightarrow 2^{X}$ is inward if $T x \subset I_{C}(x)$, for all $x \in C$.

Let $\bar{I}_{C}(x)=x+\{\lambda(z-x): z \in C, \lambda \geq 1\}$. Note that for a convex set $C$, we have $\bar{I}_{C}(x)=$ $\overline{I_{C}(x)}$. The map $T$ is weakly inward if $T x \subset \bar{I}_{C}(x)$, for all $x \in C$.

Theorem 2.1 (cf. [4]). Let $X$ be a Banach space and let $\phi \neq C \subset X$ be closed bounded convex. Let $T: C \rightarrow 2^{X}$ be upper semicontinuous $\gamma$-condensing with closed convex values, where $\gamma(\cdot)=\alpha(\cdot)$ or $\chi(\cdot)$. If $T x \bigcap \overline{I_{C}(x)} \neq \phi$ for all $x \in C$, then $T$ has a fixed point.

Lemma 2.2 [10]. Let $C$ be a nonempty closed convex subset of a Banach space $X$ and $T$ : $C \rightarrow K(X)$ a contraction. If $T x \subset \overline{I_{C}(x)}$, for all $x \in C$, then $T$ has a fixed point.

Definition 2.3. Let $C$ be a nonempty bounded closed subset of a Banach space $X$ and $\left\{x_{n}\right\}$ a bounded sequence in $X$. The asymptotic radius and the asymptotic center of $\left\{x_{n}\right\}$ in $C$ are denoted and defined by, respectively,

$$
\begin{aligned}
& r\left(C,\left\{x_{n}\right\}\right)=\inf \left\{\limsup _{n \rightarrow \infty}\left\|x_{n}-x\right\|: x \in C\right\}, \\
& A\left(C,\left\{x_{n}\right\}\right)=\left\{x \in C: \limsup _{n \rightarrow \infty}\left\|x_{n}-x\right\|=r\left(C,\left\{x_{n}\right\}\right)\right\} .
\end{aligned}
$$

If $D$ is a bounded subset of $X$, the Chebyshev radius of $D$ relative to $C$ is

$$
r_{C}(D)=\inf \{\sup \{\|x-y\|: y \in D\}: x \in C\} .
$$

Remark 2.4. The convexity of $C$ implies that $A\left(C,\left\{x_{n}\right\}\right)$ is convex. The set $A\left(C,\left\{x_{n}\right\}\right)$ is nonempty weakly compact if $C$ is weakly compact, or $C$ is a closed convex subset of a reflexive Banach space $X$ (see [5]).

Let $C$ be a nonempty bounded closed subset of a Banach space $X$. Then $\left\{x_{n}\right\}$ in $X$ is called regular with respect to $C$ if $r\left(C,\left\{x_{n}\right\}\right)=r\left(C,\left\{x_{n_{i}}\right\}\right)$ for all subsequences $\left\{x_{n_{i}}\right\}$ of $\left\{x_{n}\right\}$; while $\left\{x_{n}\right\}$ is called asymptotically uniform with respect to $C$ if $A\left(C,\left\{x_{n}\right\}\right)=$ $A\left(C,\left\{x_{n_{i}}\right\}\right)$ for all subsequences $\left\{x_{n_{i}}\right\}$ of $\left\{x_{n}\right\}$.

Lemma 2.5 [9]. Let $\left\{x_{n}\right\}$ and $C$ be as in the above definition. Then (i) there always exists a subsequence of $\left\{x_{n}\right\}$ which is regular with respect to $C$; (ii) if $C$ is separable, then $\left\{x_{n}\right\}$ contains a subsequence which is asymptotically uniform with respect to $C$.

Let $A$ be a set and $B \subset A$. A net $\left\{x_{\alpha}: \alpha \in D\right.$ (directed set) $\}$ in $A$ is eventually in $B$ if there exists $\alpha_{0} \in D$ such that $x_{\alpha} \in B$ for all $\alpha \geq \alpha_{0}$. A net $\left\{x_{\alpha}: \alpha \in D\right\}$ in a set $A$ is called an ultranet if either $\left\{x_{\alpha}: \alpha \in D\right\}$ is eventually in $B$ or $\left\{x_{\alpha}: \alpha \in D\right\}$ is eventually in $A \backslash B$, for each subset $B$ of $A$. It is well known that every net in a set has a subnet which is an ultranet (cf. [5]).

A sequence $\left\{x_{n}\right\}$ is called asymptotically $T$-regular if $\lim _{n \rightarrow \infty} d\left(x_{n}, T x_{n}\right)=0$.

Definition 2.6 [12]. Let $C$ be a nonempty weakly compact convex subset of a Banach space $X$ and $T: C \rightarrow K C(X)$. The map $T$ is called subsequentially limit contractive $(S L)$ if for 
every asymptotically $T$-regular sequence $\left\{x_{n}\right\}$ in $C$,

$$
\limsup _{n \rightarrow \infty} H\left(T x_{n}, T x\right) \leq \limsup _{n \rightarrow \infty}\left\|x_{n}-x\right\|, \quad \forall x \in A\left(C,\left\{x_{n}\right\}\right) .
$$

Every nonexpansive map is an $S L$ map but the converse does not hold (see [12, Example 3.2]).

Let $\left(\Omega, \sum\right)$ be a measurable space $\left(\sum\right.$ - $\sigma$-algebra $)$ and $C$ a subset of a metric space $X$. Then (i) $T: \Omega \rightarrow 2^{X}$ is measurable if, for any open subset $B$ of $X, T^{-1}(B) \in \sum$ where $T^{-1}(B)=\{\omega \in \Omega: T(\omega) \bigcap B \neq \phi\}$; (ii) $x: \Omega \rightarrow X$ is a measurable selector of $T: \Omega \rightarrow 2^{X}$ if $x(\cdot)$ is measurable and $x(\omega) \in T(\omega)$ for all $\omega \in \Omega$; (iii) $T: \Omega \times C \rightarrow 2^{X}$ is a random operator if, for each fixed $x \in C, T(\cdot, x): \Omega \rightarrow 2^{X}$ is measurable; (iv) $x: \Omega \rightarrow C$ is a random fixed point of $T: \Omega \times C \rightarrow 2^{X}$ if $x$ is measurable and $x(\omega) \in T(\omega, x(\omega))$, for each $\omega \in \Omega$; (v) $T: \Omega \times C \rightarrow 2^{X}$ is continuous (resp., contractive, nonexpansive, $S L$, etc.) if for each $\omega \in \Omega, T(\omega, \cdot)$ is continuous (resp., contractive, nonexpansive, $S L$, etc.).

We will denote by $F(\omega)$ the fixed point set of $T(\omega, \cdot)$, that is, $F(\omega)=\{x \in C: x \in$ $T(\omega, x)\}$.

We list the following results for a ready reference in the sequel.

Lemma 2.7 [15]. Let $(X, d)$ be a complete separable metric space and $T: \Omega \rightarrow C(X)$ a measurable operator. Then $T$ has a measurable selector.

Lemma 2.8 [6]. Suppose that $\left\{T_{n}\right\}$ is a sequence of measurable multivalued operators from $\Omega$ to $C B(X)$ and $T: \Omega \rightarrow C B(X)$ is an operator. If, for each $\omega \in \Omega, H\left(T_{n}(\omega), T(\omega)\right) \rightarrow 0$, then $T$ is measurable.

Lemma 2.9 [14]. Let $X$ be a separable metric space and $Y$ a metric space. If $f: \Omega \times X \rightarrow Y$ is measurable in $\omega \in \Omega$ and continuous in $x \in X$, and if $x: \Omega \rightarrow X$ is measurable, then $f(\cdot, x(\cdot)): \Omega \rightarrow Y$ is measurable.

Lemma 2.10 [11, Lemma 2.4]. Let $C$ be a closed separable subset of a Banach space $X, T$ : $\Omega \times C \rightarrow C$ a random continuous operator, and $F: \Omega \rightarrow 2^{C}$ a measurable closed-valued operator. Then for any $s>0$, the operator $G: \Omega \rightarrow 2^{C}$ given by $G(\omega)=\{x \in F(\omega): \| x-$ $T(\omega, x) \|<s\}, \omega \in \Omega$, is measurable and so is the operator $\operatorname{cl}\{G(\omega)\}$ (the closure of $G(\omega)$ ).

\section{Main results}

The following lemma is proved, as a claim, in the proof of [12, Theorem 3.3].

Lemma 3.1. Let $C$ be a nonempty closed bounded convex subset of a Banach space $X$ and $T: C \rightarrow K C(X)$ an SL map such that Tx $\subset I_{C}(x)$, for all $x \in C$. If $\left\{x_{n}\right\}$ is asymptotically $T$-regular in $C$, then there exists an ultranet $\left\{x_{n_{\alpha}}\right\}$ of $\left\{x_{n}\right\}$ such that $T x \cap I_{A}(x) \neq \phi$, for all $x \in A$, where $A=A\left(C,\left\{x_{n_{\alpha}}\right\}\right)$.

We now set out to establish a random fixed point result which, on the one hand, is a stochastic version of [12, Theorem 3.3] (which itself extends [5, Theorem 3.4]), and on the other hand, improves [11, Theorem 3.3]. We follow the arguments of the proof of [11, Theorem 3.2]. 
Theorem 3.2. Let $C$ be a nonempty closed bounded convex separable subset of a Banach space $X$ with $\epsilon_{\alpha}(X)<1$ and $T: \Omega \times C \rightarrow K C(X)$, a continuous $1-\chi$-contractive and SL random operator. If $T(\omega, x) \subset I_{C}(x)$, for all $x \in C$ and $\omega \in \Omega$, then $T$ has a random fixed point.

Proof. Let $x_{0} \in C$ be fixed and consider the measurable map $x_{0}(\omega)=x_{0}$, for all $\omega \in \Omega$. For each $n \geq 1$, define $T_{n}(\omega, \cdot): C \rightarrow K C(X)$ by

$$
T_{n}(\omega, x)=\frac{1}{n} x_{0}(\omega)+\left(1-\frac{1}{n}\right) T(\omega, x), \quad \forall x \in C
$$

Then $T_{n}$ is contractive and $T_{n}(\omega, x) \subset I_{C}(x)$, for all $x \in C$. Hence, by Lemma 2.2, each $T_{n}$ has a deterministic fixed point $z_{n}(\omega) \in C$. So,

$$
d\left(z_{n}(\omega), T\left(\omega, z_{n}(\omega)\right)\right) \leq \frac{1}{n} \operatorname{diam} C \longrightarrow 0 \quad \text { as } n \longrightarrow \infty .
$$

Thus

$$
F_{n}(\omega)=\left\{x \in C: d(x, T(\omega, x)) \leq \frac{1}{n} \operatorname{diam} C\right\}
$$

is nonempty closed and convex. By Lemma 2.10, each $F_{n}$ is measurable. Thus, by Lemma 2.7, $F_{n}$ admits a measurable selector $x_{n}(\omega)$ such that

$$
d\left(x_{n}(\omega), T\left(\omega, x_{n}(\omega)\right)\right) \leq \frac{1}{n} \operatorname{diam} C \longrightarrow 0 \quad \text { as } n \longrightarrow \infty
$$

Define $f: \Omega \times C \rightarrow[0, \infty)$ by

$$
f(\omega, x)=\lim _{n \rightarrow \infty} \sup \left\|x_{n}(\omega)-x\right\|, \quad x \in C .
$$

It is easy to see that $f(\omega, \cdot)$ is continuous and $f(\cdot, x)$ is measurable, so by Lemma 2.9, $f(\cdot, x)$ is measurable. Obviously, $f(\omega, \cdot)$ is convex. Therefore, by [13, Lemma 1.3.9], it is weakly lower semicontinuous. Note that $\epsilon_{\alpha}(X)<1$, so $X$ is reflexive. Therefore, $C$ is weakly compact. Hence, by [3, Theorem 3.4], the marginal function $r(\omega)=\inf _{x \in C} f(\omega, x)$ and the marginal map $A(\omega)=\{x \in C: f(\omega, x)=r(\omega)\}$ are measurable. By Remark 2.4, $A(\omega)$ is a weakly compact convex subset of $C$. For any $\omega \in \Omega$, we may assume that the sequence $\left\{x_{n_{\alpha}}(\omega)\right\}$ is an ultranet in $C$. Note that $A(\omega)=A\left(C,\left\{x_{n_{\alpha}}(\omega)\right\}\right)$ and $r(\omega)=$ $r\left(C,\left\{x_{n_{\alpha}}(\omega)\right\}\right)$. We now apply [5, Theorem 3.2] to obtain

$$
r_{C}(A(\omega)) \leq \lambda r\left(C,\left\{x_{n_{\alpha}}(\omega)\right\}\right),
$$

where $\lambda=1-\triangle_{X, \alpha}\left(1^{-}\right)<1$.

For each $\omega \in \Omega$ and $n \geq 1$, we define the multivalued contraction $T_{n}^{1}(\omega, \cdot): A(\omega) \rightarrow$ $K C(X)$ by

$$
T_{n}^{1}(\omega, x)=\frac{1}{n} x_{1}(\omega)+\left(1-\frac{1}{n}\right) T(\omega, x), \quad \text { for each } x \in C .
$$


6 Random fixed points of multivalued inward random operators

By Lemma 3.1, we have

$$
T(\omega, x) \bigcap I_{A(\omega)}(x) \neq \phi, \quad \forall x \in A(\omega) .
$$

Since $I_{A(\omega)}(x)$ is convex, it follows, from (3.7) and (3.8), that

$$
T_{n}^{1}(\omega, x) \bigcap I_{A(\omega)}(x) \neq \phi, \quad \forall x \in A(\omega)
$$

As in the proof of $\left[4\right.$, Proposition 4.1], we can prove that $T_{n}^{1}(\omega, \cdot)$ is $\chi$-condensing. Hence, by Theorem $2.1, T_{n}^{1}(\omega, \cdot)$ has a fixed point $z_{n}^{1}(\omega) \in A(\omega)$; that is, $F(\omega) \cap A(\omega) \neq \phi$. Clearly,

$$
d\left(z_{n}^{1}(\omega), T\left(\omega, z_{n}^{1}(\omega)\right)\right) \leq \frac{1}{n} \operatorname{diam} C \longrightarrow 0 \quad \text { as } n \longrightarrow \infty .
$$

Thus

$$
F_{n}^{1}(\omega)=\left\{x \in A(\omega): d(x, T(\omega, x)) \leq \frac{1}{n} \operatorname{diam} C\right\}
$$

is nonempty closed and convex for each $n \geq 1$. By Lemma 2.10, each $F_{n}^{1}$ is measurable. So, by Lemma 2.7 we can choose $x_{n}^{1}$ a measurable selector of $F_{n}^{1}$. Thus we have $x_{n}^{1} \in A(\omega)$ and $d\left(x_{n}^{1}(\omega), T\left(\omega, x_{n}^{1}(\omega)\right)\right) \rightarrow 0$ as $n \rightarrow \infty$. Let $f_{2}: \Omega \times C \rightarrow[0, \infty)$ be defined by

$$
f_{2}(\omega, x)=\lim _{n \rightarrow \infty} \sup \left\|x_{n}^{1}(\omega)-x\right\|, \quad \forall \omega \in \Omega .
$$

As above, $f_{2}$ is measurable and weakly lower semicontinuous. Again, by [3, Theorem 3.4], $r_{2}(\omega)=\inf _{x \in A(\omega)} f_{2}(\omega, x)$ and $A^{1}(\omega)=\left\{x \in A(\omega): f_{2}(\omega, x)=r_{2}(\omega)\right\}$ are measurable. Since $A^{1}(\omega)=A\left(A(\omega),\left\{x_{n_{\alpha}}^{1}(\omega)\right\}\right)$, it follows that $A^{1}(\omega)$ is weakly compact and convex. We also note that $r_{2}(\omega)=r\left(A(\omega),\left\{x_{n_{\alpha}}^{1}(\omega)\right\}\right)$. Again, for any $\omega \in \Omega$, we can assume that the sequence $\left\{x_{n_{\alpha}}^{1}(\omega)\right\}_{\alpha}$ is an ultranet in $A^{1}(\omega)$. As above, by Lemma 3.1 and [5, Theorem 3.2], we obtain

$$
T(\omega, x(\omega)) \bigcap I_{A^{1}(\omega)}(x(\omega)) \neq \phi, \quad \forall x(\omega) \in A^{1}(\omega),
$$

where $A^{1}(\omega)=A\left(A(\omega),\left\{x_{n_{\alpha}}^{1}(\omega)\right\}\right)$ and

$$
r_{C}\left(A^{1}(\omega)\right) \leq \lambda r\left(A(\omega),\left\{x_{n_{\alpha}}^{1}(\omega)\right\}\right) \leq \lambda r_{C}(A(\omega)) .
$$

By induction, for each $m \geq 1$, we take a sequence $\left\{x_{n}^{m}(\omega)\right\}_{n} \subset A^{m-1}$ such that

$$
\lim _{n \rightarrow \infty} d\left(x_{n}^{m}(\omega), T\left(\omega, x_{n}^{m}(\omega)\right)\right)=0
$$

for each $\omega \in \Omega$. By means of the ultranet $\left\{x_{n_{\alpha}}^{m}(\omega)\right\}_{\alpha}$, we construct the set $A^{m}=A(C$, $\left.\left\{x_{n_{\alpha}}^{m}(\omega)\right\}\right)$ such that $r_{C}\left(A^{m}\right) \leq \lambda^{m} r_{C}(A)$. Since diam $A^{m}(\omega) \leq 2 r_{C}\left(A^{m}\right)$ and $\lambda<1$, it follows that $\lim _{m \rightarrow \infty} \operatorname{diam} A^{m}(\omega)=0$. Note that $\left\{A^{m}(\omega)\right\}$ is a descending sequence of weakly compact subsets of $C$ for each $\omega \in \Omega$. Thus, by Cantor's intersection theorem (see [ 1 , page 20]), we have $\bigcap_{m} A^{m}(\omega)=\{z(\omega)\}$ for some $z(\omega) \in C$. Furthermore, we observe that 
$H\left(A^{m}(\omega),\{z(\omega)\}\right) \leq \operatorname{diam} A^{m}(\omega) \rightarrow 0$ as $m \rightarrow \infty$. Therefore, by Lemma $2.8, z(\omega)$ is measurable. Finally, we show that $z(\omega)$ is a random fixed point of $T$. For each $m \geq 1$, we have

$$
\begin{aligned}
d(z(\omega), T(\omega, z(\omega))) \leq & \left\|z(\omega)-x_{n}^{m}(\omega)\right\|+d\left(x_{n}^{m}(\omega), T\left(\omega, x_{n}^{m}(\omega)\right)\right) \\
& +H\left(T\left(\omega, x_{n}^{m}(\omega)\right), T(\omega, z(\omega))\right) .
\end{aligned}
$$

Since $T$ is an $S L$ map and $\left\{x_{n}^{m}(\omega)\right\}$ asymptotically $T$-regular for $m \geq 1$, therefore we have

$$
\lim _{n \rightarrow \infty} \sup H\left(T\left(\omega, x_{n}^{m}(\omega), T(\omega, z(\omega))\right)\right) \leq \lim _{n \rightarrow \infty} \sup \left\|x_{n}^{m}(\omega)-z(\omega)\right\|
$$

Thus, by (3.15)-(3.17), we obtain

$$
d(z(\omega), T(\omega, z(\omega))) \leq 2 \lim _{n \rightarrow \infty} \sup \left\|z(\omega)-x_{n}^{m}(\omega)\right\| \leq 2 \operatorname{diam} A^{m}(\omega)
$$

Taking the limit as $m \rightarrow \infty$, we have $z(\omega) \in T(\omega, z(\omega))$.

We remark that if $C$ is a weakly compact subset of a reflexive Banach space satisfying the nonstrict Opial's property, then we can follow the ideas in the proof of [4, Theorem 4.5] to deduce that a nonexpansive map $T: C \rightarrow K(X)$ with bounded range is 1- $\chi$ contractive. Thus, in view of Theorem 3.2, we have the following.

Corollary 3.3. Let $X$ be a Banach space satisfying the nonstrict Opial's property and $\epsilon_{\alpha}(X)$ $<1$. Suppose that $C$ is a nonempty closed bounded convex separable subset of $X$ and $T$ : $\Omega \times C \rightarrow K C(X)$ is a nonexpansive random operator such that $T(C)$ is a bounded set and $T(\omega, x) \subset I_{C}(x)$, for all $x \in C$ and $\omega \in \Omega$. Then $T$ has a random fixed point.

Remark 3.4. The ultranet in Lemma 3.1 can be replaced by a sequence which is asymptotically uniform with respect to $C$ (see [9]). This allows us to rewrite the proof of Theorem 3.2 for $\beta$ and $\chi$ moduli of noncompact convexity.

The following two theorems follow from the above remark and [4, Theorem 3.4].

THeOrem 3.5. Let $C$ be a nonempty closed bounded convex separable subset of a Banach space $X$ with $\epsilon_{\beta}(X)<1$ and $T: \Omega \times C \rightarrow K C(X)$, continuous 1- $\chi$-contractive, and SL random operator. If $T$ satisfies the inwardness condition, then $T$ has a random fixed point.

Theorem 3.6. Let $X$ be a Banach space satisfying the nonstrict Opial's property and $\epsilon_{\chi}(X)<$ 1. Suppose that $C$ is a nonempty closed bounded convex separable subset of $X$ and $T: \Omega \times$ $C \rightarrow K C(X)$ is a nonexpansive random operator such that $T(C)$ is a bounded set and $T$ satisfies the inwardness condition. Then $T$ has a random fixed point.

\section{Acknowledgment}

The authors gratefully acknowledge the support provided by the King Fahd University of Petroleum \& Minerals during this research. 
8 Random fixed points of multivalued inward random operators

\section{References}

[1] J. M. Ayerbe Toledano, T. Domínguez Benavides, and G. López Acedo, Measures of Noncompactness in Metric Fixed Point Theory, Operator Theory: Advances and Applications, vol. 99, Birkhäuser, Basel, 1997.

[2] K. Deimling, Nonlinear Functional Analysis, Springer, Berlin, 1985.

[3] T. Domínguez Benavides, G. López Acedo, and H. K. Xu, Random fixed points of set-valued operators, Proceedings of the American Mathematical Society 124 (1996), no. 3, 831-838.

[4] T. Domínguez Benavides and P. Lorenzo Ramírez, Fixed-point theorems for multivalued nonexpansive mappings without uniform convexity, Abstract and Applied Analysis 2003 (2003), no. 6, $375-386$.

[5] _ Fixed point theorems for multivalued nonexpansive mappings satisfying inwardness conditions, Journal of Mathematical Analysis and Applications 291 (2004), no. 1, 100-108.

[6] S. Itoh, A random fixed point theorem for a multivalued contraction mapping, Pacific Journal of Mathematics 68 (1977), no. 1, 85-90.

[7] W. A. Kirk and S. Massa, Remarks on asymptotic and Chebyshev centers, Houston Journal of Mathematics 16 (1990), no. 3, 357-364.

[8] T. Kuczumow and S. Prus, Compact asymptotic centers and fixed points of multivalued nonexpansive mappings, Houston Journal of Mathematics 16 (1990), no. 4, 465-468.

[9] T.-C. Lim, Remarks on some fixed point theorems, Proceedings of the American Mathematical Society 60 (1976), no. 1, 179-182.

[10] _ A fixed point theorem for weakly inward multivalued contractions, Journal of Mathematical Analysis and Applications 247 (2000), no. 1, 323-327.

[11] S. Plubtieng and P. Kumam, Random fixed point theorems for multivalued nonexpansive non-selfrandom operators, Journal of Applied Mathematics and Stochastic Analysis 2006 (2006), Article ID 43796, 9 pages.

[12] N. Shahzad and A. Lone, Fixed points of multimaps which are not necessarily nonexpansive, Fixed Point Theory and Applications 2005 (2005), no. 2, 169-176.

[13] W. Takahashi, Nonlinear Functional Analysis, Yokohama, Yokohama, 2000.

[14] K.-K. Tan and X.-Z. Yuan, Some random fixed point publishers, Fixed Point Theory and Applications (Halifax, NS, 1991) (K.-K. Tan, ed.), World Scientific, New Jersey, 1992, pp. 334-345.

[15] D. H. Wagner, Survey of measurable selection theorems, SIAM Journal on Control and Optimization 15 (1977), no. 5, 859-903.

A. R. Khan: Department of Mathematical Sciences, King Fahd University of Petroleum \& Minerals, Dhahran 31261, Saudi Arabia

E-mail address: arahim@kfupm.edu.sa

A. A. Domlo: Department of Mathematical Sciences, King Fahd University of Petroleum \& Minerals, Dhahran 31261, Saudi Arabia

E-mail address: g20052@kfupm.edu.sa 


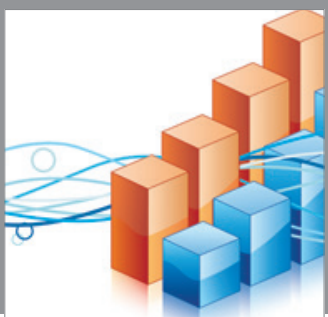

Advances in

Operations Research

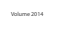

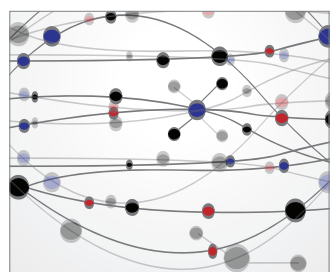

\section{The Scientific} World Journal
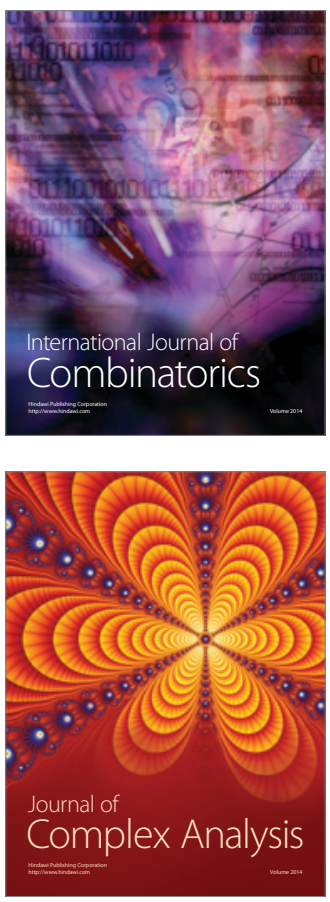

International Journal of

Mathematics and

Mathematical

Sciences
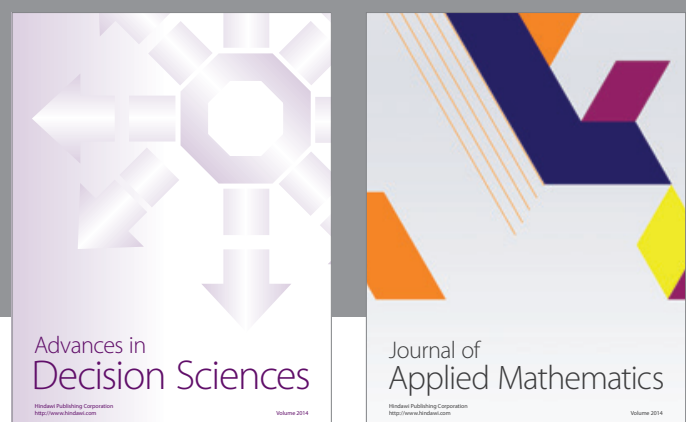

Journal of

Applied Mathematics
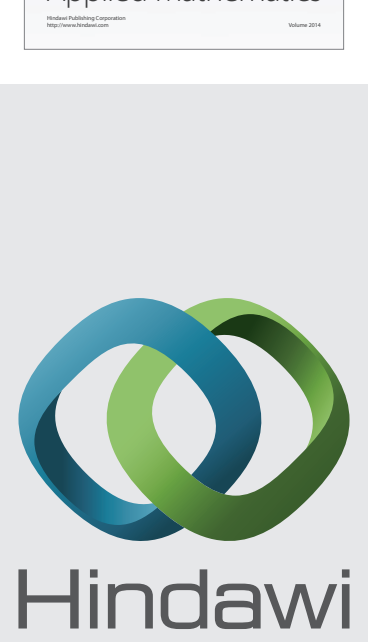

Submit your manuscripts at http://www.hindawi.com
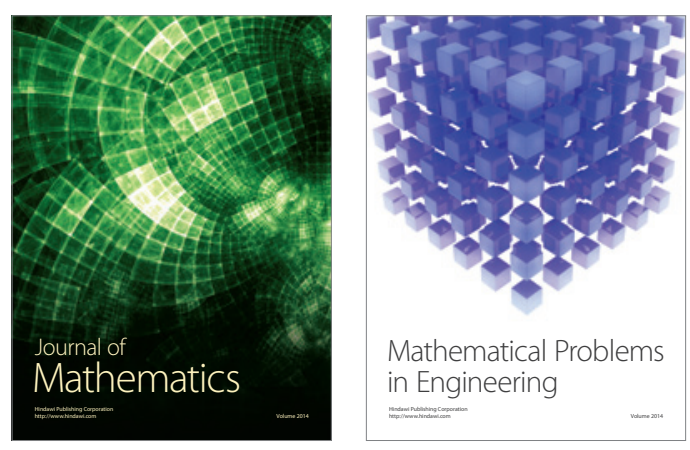

Mathematical Problems in Engineering
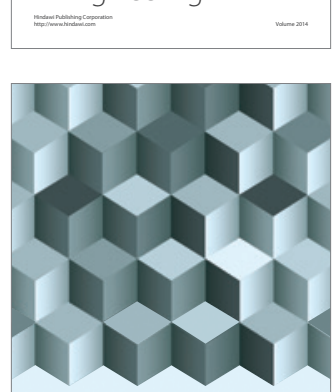

Journal of

Function Spaces
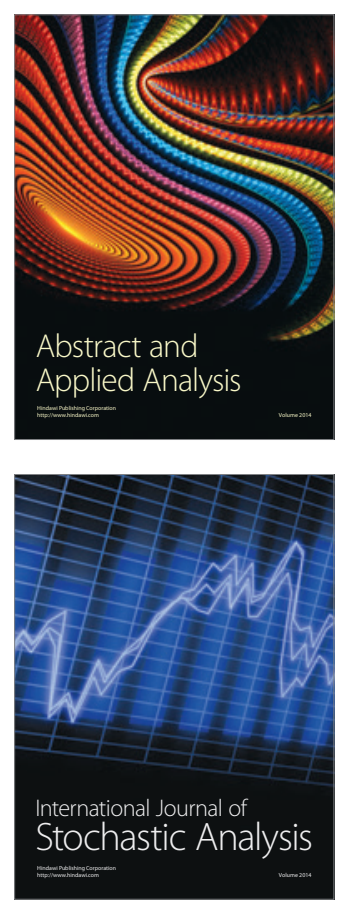

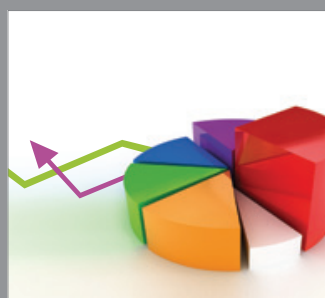

ournal of

Probability and Statistics

Promensencen
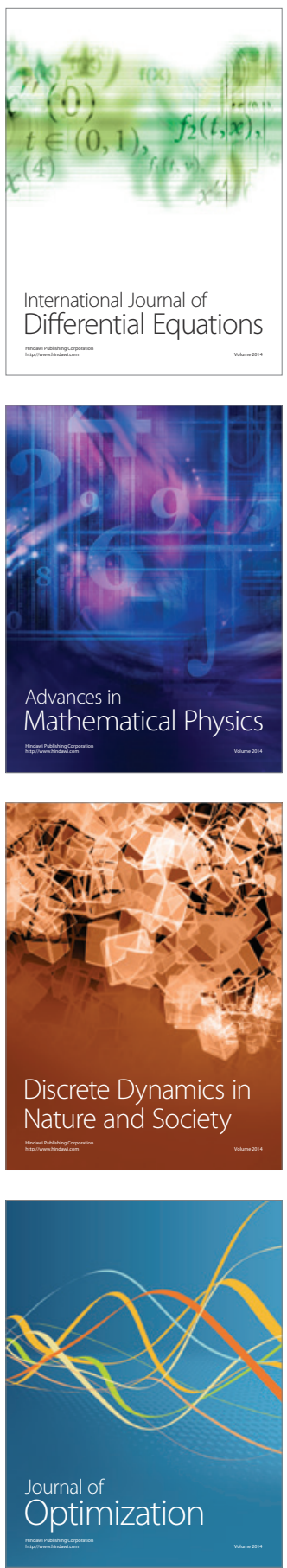